University of Nebraska - Lincoln

DigitalCommons@University of Nebraska - Lincoln

Faculty Publications: Materials Research

Science and Engineering Center

Materials Research Science and Engineering

Center

5-9-2003

Resonant Inversion of Tunneling Magnetoresistance

Evgeny Y. Tsymbal

University of Nebraska - Lincoln, tsymbal@unl.edu

Andrei Sokolov

University of Nebraska-Lincoln, sokolov@unl.edu

Ildar F. Sabiryanov

University of Nebraska - Lincoln, isabirianov2@unl.edu

Bernard Doudin

University of Nebraska-Lincoln, bernard.doudin@ipcms.unistra.fr

Follow this and additional works at: https://digitalcommons.unl.edu/mrsecfacpubs

Part of the Materials Science and Engineering Commons

Tsymbal, Evgeny Y.; Sokolov, Andrei; Sabiryanov, Ildar F.; and Doudin, Bernard, "Resonant Inversion of Tunneling Magnetoresistance" (2003). Faculty Publications: Materials Research Science and Engineering Center. 55.

https://digitalcommons.unl.edu/mrsecfacpubs/55

This Article is brought to you for free and open access by the Materials Research Science and Engineering Center at DigitalCommons@University of Nebraska - Lincoln. It has been accepted for inclusion in Faculty Publications:

Materials Research Science and Engineering Center by an authorized administrator of DigitalCommons@University of Nebraska - Lincoln. 


\title{
Resonant Inversion of Tunneling Magnetoresistance
}

\author{
E. Y. Tsymbal, A. Sokolov, I. F. Sabirianov, and B. Doudin \\ Department of Physics and Astronomy, University of Nebraska-Lincoln, Lincoln, Nebraska 68588-0111, USA
}

(Received 7 July 2002; published 8 May 2003)

\begin{abstract}
Resonant tunneling via localized states in the barrier can invert magnetoresistance in magnetic tunnel junctions. Experiments performed on electrodeposited $\mathrm{Ni} / \mathrm{NiO} / \mathrm{Co}$ nanojunctions of area smaller than $0.01 \mu \mathrm{m}^{2}$ show that both positive and negative values of magnetoresistance are possible. Calculations based on Landauer-Büttiker theory explain this behavior in terms of disorder-driven statistical variations in magnetoresistance with a finite probability of inversion due to resonant tunneling.
\end{abstract}

DOI: 10.1103/PhysRevLett.90.186602

PACS numbers: 72.25.-b, 73.23.-b, 73.40.Rw, 73.63.Rt

Magnetic tunnel junctions (MTJs) made of two ferromagnetic electrodes separated by an insulating spacer layer have aroused considerable interest due to potential applications in spin-electronic devices such as magnetic sensors and magnetic random access memories. Functioning of these devices is controlled by the phenomenon of tunneling magnetoresistance (TMR), the essence of which is a dramatic change in the tunneling current when relative magnetizations of the two ferromagnetic layers change their alignment (for recent reviews see Ref. [1]). Within the simplest model, the magnitude of TMR is determined solely by the spin polarization (SP) of the density of states at the Fermi energy of the two ferromagnets, $P_{1}$ and $P_{2}[2,3]$, so that

$$
\mathrm{TMR} \equiv \frac{G_{P}-G_{A P}}{G_{P}+G_{A P}}=P_{1} P_{2} .
$$

Here, $G_{P}$ and $G_{A P}$ are the conductance for the parallel and antiparallel alignment of the MTJ, and we use the definition of TMR similar to that of Ref. [3]. If both ferromagnets have the same sign of the SP, the conductance is larger when the two magnetic layers are aligned parallel. This is what is generally observed [1] and referred to as the normal (positive) sign of TMR. More sophisticated models show that TMR depends strongly on the atomic and electronic structure of the entire MTJ, which includes the insulator and the ferromagnet/ insulator interfaces. In particular, as was predicted theoretically, the TMR is sensitive to the height [4] and the shape [5] of the potential barrier, to the degree of disorder within the barrier [6], to the bonding at the ferromagnet/ insulator interfaces [7], and to the electronic structure of the insulator [8,9]. Experimentally, it was found that impurities in the barrier [10] and electronic structure of the interfaces [11] strongly affect the TMR.

The most remarkable effect of the insulator is the inversion of the SP of tunneling electrons. De Teresa et al. found a negative SP of Co using a $\mathrm{SrTiO}_{3}$ barrier [12]. This behavior is opposite to what is known for alumina spacers for which all ferromagnetic $3 d$ metals display positive SP [13]. The inversion of the SP observed in these experiments was attributed to the effect of bonding at the ferromagnet/barrier interface that had been predicted theoretically in Ref. [7]. The same mechanism was put forward to explain positive and negative values of TMR depending on the applied voltage in MTJs with $\mathrm{Ta}_{2} \mathrm{O}_{5}$ and $\mathrm{Ta}_{2} \mathrm{O}_{5} / \mathrm{Al}_{2} \mathrm{O}_{3}$ barriers [14].

In this Letter, we show that a different mechanism for the inversion of TMR can occur. Tunneling via localized states in the barrier under resonant conditions leads to a change in sign of the effective spin polarization of conducting electrons. Magnetic nanojunctions of cross section smaller than $0.01 \mu \mathrm{m}^{2}$ grown by electrodeposition [15] exhibit magnetoresistive properties that illustrate this model. By performing measurements of TMR involving many samples and comparing experimental and calculated statistics, we confirm that the TMR is inverted when the energy of a localized state in the barrier matches the Fermi energy of the ferromagnetic electrodes.

Samples were synthesized using electrochemical techniques. Polyester track-etched membranes, $6 \pm 1 \mu \mathrm{m}$ thick, with cylindrical holes of $80 \pm 20 \mathrm{~nm}$ diameter, were used as templates. The membrane was half filled by electrodeposition of $\mathrm{Ni}$ in the holes. A film of $\mathrm{NiO}$ was subsequently obtained by anodization process. Characterization of the dielectric layer properties has been performed by means of impedance spectroscopy [16]. The estimated thickness of $\mathrm{NiO}$ is about $1.5 \mathrm{~nm}$. MottSchottky analysis gives an estimate for the carrier density at ambient temperature, $n=10^{25} \mathrm{~m}^{-3}$, one order of magnitude smaller than previously reported [17]. The top ferromagnet was made by Co electrodeposition in a nonaqueous bath, avoiding the dissolution of the oxide film. Details of the procedure were published elsewhere $[15,16]$.

Electrical properties of the $\mathrm{Ni} / \mathrm{NiO} / \mathrm{Co}$ nanowires were investigated at $1.6-4.2 \mathrm{~K}$ using dc techniques. The estimated resistance value of a $\mathrm{Ni} / \mathrm{Co}$ metallic wire is $1-3 \mathrm{k} \Omega$. Samples with resistance values smaller than a 
few tens of $\mathrm{k} \Omega$ were classified as short, and discarded. Samples with resistance larger than a few tens of $M \Omega$, showing very noisy features, were also discarded. Among over 200 samples fabricated, 87 were fulfilling the required resistance range conditions and therefore were included in the presented results. The measured distribution of TMR values is displayed in Fig. 1 on two scales. One is the TMR defined according to Eq. (1), and the other is the commonly used TMR ratio, $\left(R_{A P}-R_{P}\right) / R_{P}$, where $R_{P}$ and $R_{A P}$ are the resistance for the parallel and antiparallel alignment, respectively. As is evident from Fig. 1, the distribution is very broad with the highest peak lying at positive values close to zero. Some tunnel junctions display very large values of TMR up to 0.17 (40\% if we use the standard definition of TMR). The remarkable fact is the appearance of the inverse magnetoresistance observed on ten samples. The largest negative value was found to be -0.14 ( $-25 \%$ using the standard definition).

The insets of Fig. 1 display magnetoresistance curves measured on the samples displaying the largest positive and negative values of TMR. The sharpness of the magnetoresistance curves indicates unambiguously that a single wire is connected. If two or more wires are measured in parallel, we expect to observe several steps in the magnetoresistance curve, corresponding to different magnetic switching fields of different wires [18]. This might be the case for the samples with low TMR values (less than 0.01). In this case, multiple parallel connections are hindered by the measurement noise. Therefore, our statistics displayed in Fig. 1 might also include junctions of a larger area, which could cause a sharp peak in the distribution at positive values close to zero (indicated by the unshaded bar).

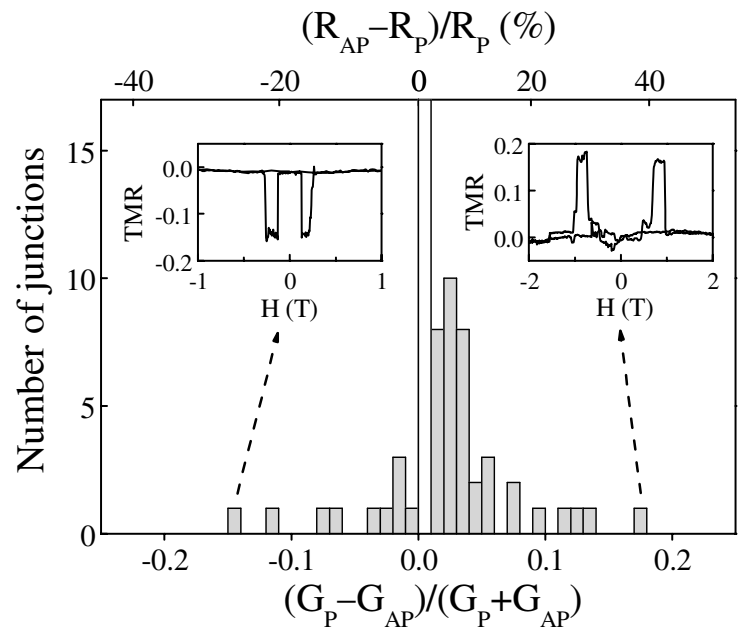

FIG. 1. Histogram of the magnetoresistance distribution in magnetic $\mathrm{Ni} / \mathrm{NiO} / \mathrm{Co}$ nanojunctions. The vertical scale is cut at $N=17$ (at the highest peak $N=39$ ). The unshaded bar indicates a possible contribution from samples with multiple junctions. Insets show magnetoresistance curves for the highest positive and negative values of TMR observed.
The inversion of TMR observed in our experiments can be understood within a simple one-dimensional picture of tunneling via an impurity state in the barrier. In this case, the conductance as a function of energy $E$ is given by

$$
G(E)=\frac{4 e^{2}}{h} \frac{\Gamma_{1} \Gamma_{2}}{\left(E-E_{i}\right)^{2}+\left(\Gamma_{1}+\Gamma_{2}\right)^{2}},
$$

where $E_{i}$ is the energy of the impurity state, and $\Gamma_{1} / \hbar$ and $\Gamma_{2} / \hbar$ are leak rates of an electron from the impurity state to the left and right electrodes. We assume for simplicity that the latter are proportional to the densities of states (DOS) of the electrodes, $\rho_{1}$ and $\rho_{2}$, at the left and right interfaces, so that $\Gamma_{1} \propto \rho_{1} \exp [-2 \kappa x]$ and $\Gamma_{2} \propto$ $\rho_{2} \exp [-2 \kappa(d-x)]$, where $\kappa$ is the decay constant and $x$ is the position of the impurity within the barrier of thickness $d$. Off resonance, when $\left|E-E_{i}\right| \gg \Gamma_{1}+\Gamma_{2}$, the latter assumption implies that the spin conductance is given by $G \propto \rho_{1} \rho_{2}$. When tunneling occurs between ferromagnetic electrodes this leads to TMR, which is given by Eq. (1) with $P_{1,2}=\left(\rho_{1,2}^{\uparrow}-\rho_{1,2}^{\downarrow}\right) /\left(\rho_{1,2}^{\uparrow}+\rho_{1,2}^{\downarrow}\right)$. At resonance, when $E=E_{i}$, the situation is different. Assuming for simplicity an asymmetric position of impurity, we obtain from Eq. (2) that $G \propto \rho_{2} / \rho_{1}$, if $x<d / 2$ and, hence, $\Gamma_{1} \gg \Gamma_{2}$, and we obtain $G \propto \rho_{1} / \rho_{2}$, if $x>$ $d / 2$ and, hence, $\Gamma_{1} \ll \Gamma_{2}$. In both cases, the conductance is inversely proportional to the DOS of the one of the ferromagnets that results in the sign inversion:

$$
\mathrm{TMR}=-P_{1} P_{2} .
$$

Thus, the spin-dependent leak rates under resonant conditions invert the effective SP of one of the electrodes.

In order to observe this inversion of TMR, one needs to provide experimental conditions at which the energy of a localized state lies close to the $E_{F}$ and resonant tunneling dominates direct tunneling. This is the case for our smallarea tunnel junctions which display two-level fluctuations of the electric current, thereby indicating an impurity/ defect-driven transport [15]. This is, however, not the case for thin-film MTJs of a relatively large area (that spans values from a fraction of $\mu \mathrm{m}^{2}$ to a few $\mathrm{mm}^{2}$ ) in which the resultant conductance is the sum over a large number of local disorder configurations, and on the average the TMR is reduced [6]. The latter argument explains small positive TMR values of $0.01(2 \%)$ or less observed earlier in the experiments performed on large-area $\mathrm{Ni} / \mathrm{NiO} / \mathrm{Co}$ samples $[18,19]$.

The decisive role of localized states in the inversion of TMR is illustrated by the measured voltage dependence shown in Fig. 2 for three different junctions. If localized states lie far away from the Fermi energy, the TMR is positive and large, decreasing with bias, as can be seen from Fig. 2(a). Such a decrease is well known for "standard" thin-film MTJs and is most likely the consequence of spin-flip scattering [1]. If a localized state lies close to the $E_{F}$, but not close enough to invert the TMR at zero 
bias, the inversion can occur at finite bias. An example of such a behavior is demonstrated in Fig. 2(b) on a sample of a small TMR value (about 0.01) at low bias. The sign change of TMR is the consequence of a localized state which lies above the $E_{F}$, as is schematically explained in the right-hand panels of Fig. 2. At small $V$, only electrons at the $E_{F}$ tunnel through the barrier. If the impurity state has energy $E_{i}$ above $E_{F}$ and its width $\Gamma$ is less than $E_{i}-E_{F}$, then this state reduces the potential barrier height, thereby diminishing the TMR [4]. On the other hand, if $V$ becomes larger than $E_{i}-E_{F}$, the contribution from electrons tunneling via the localized state can invert the TMR. Using Eq. (2), the conductance $\int_{E_{F}}^{E_{F}+e V} G(E) d E$ can be calculated as a function of $V$ assuming for simplicity an asymmetric position of impurity and energyindependent $\rho_{1}$ and $\rho_{2}$. The solid line in Fig. 2 shows the calculated TMR for $E_{i}=35 \mathrm{meV}$ and $\Gamma=25 \mathrm{meV}$ which displays a sign change at positive bias. The drop of TMR with $V$ for negative bias is similar to that for junctions of large positive values [Fig. 2(a)], and cannot be explained by the model of Eq. (2).

The variation of TMR versus $V$ for MTJs displaying inverse TMR is controlled by the position and the width of the resonant state. In general, we observe asymmetric curves with a minimum of TMR shifted to either positive or negative bias. An example is presented in Fig. 2(c) for a MTJ with TMR $=-0.02$ at low bias. A small resonant width, $\Gamma=5 \mathrm{meV}$, results in a very abrupt change of

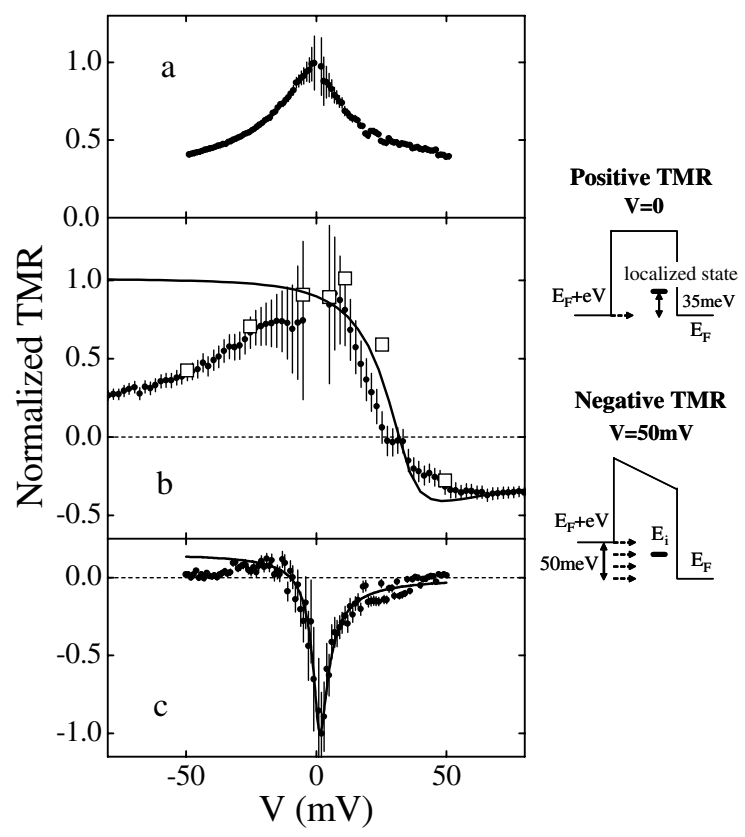

FIG. 2. Normalized magnetoresistance as a function of bias voltage for MTJs with TMR $=0.17$ (a), TMR $=0.01$ (b), and $\mathrm{TMR}=-0.02$ (c) at low bias. Experimental data are obtained from $I-V$ characteristics (dots) and from full $R(H)$ curves (squares). Solid lines represent calculated data. Right-hand panels explain schematically sign change of TMR with bias shown in Fig. 2(b).
TMR versus $V$ in this case. As predicted by Eq. (2) [solid line in Fig. 2(c)], the TMR changes sign at negative bias. Then it drops to zero due to spin-flip scattering, similar to that in Figs. 2(a) and 2(b).

A microscopic quantum model for the conduction in disordered junctions of small area allows us to go beyond the simplifications of Eq. (2), and get insight into statistics of resonant tunneling. We have calculated the TMR using Landauer-Büttiker theory [20] within a single-band tight-binding model. The barrier is represented by an $8 \times$ $8 \times 8$ atomic structure with a simple cubic geometry and (001) orientation of atomic layers. All energies are measured in units of hopping integral $\beta$ relative to $E_{F}$. A value of $\beta$ can be estimated from the bandwidth $w=$ $12 \beta$. Taking into account that the experimental width of the conduction band in $\mathrm{NiO}$ is $18 \mathrm{eV}$ [21], we find that $\beta \approx$ $1.5 \mathrm{eV}$. The on-site atomic energy of the barrier atoms is set equal to $7 \beta$. This provides no states at the $E_{F}$ in the perfect structure and simulates a tunneling barrier of height $U=7 \beta-w / 2=\beta \approx 1.5 \mathrm{eV}$. This value is of the order of half the experimental band gap in $\mathrm{NiO}$ which is about $2 \mathrm{eV}$ [21]. Disorder is introduced as a random variation of the on-site atomic energies with a uniform distribution of width $\gamma$. This disorder broadens the conduction band creating localized states within the band gap of the insulator.

For calculating the conductance, the disordered barrier layer is inserted between two ferromagnetic electrodes. The influence of the electrodes on the electronic

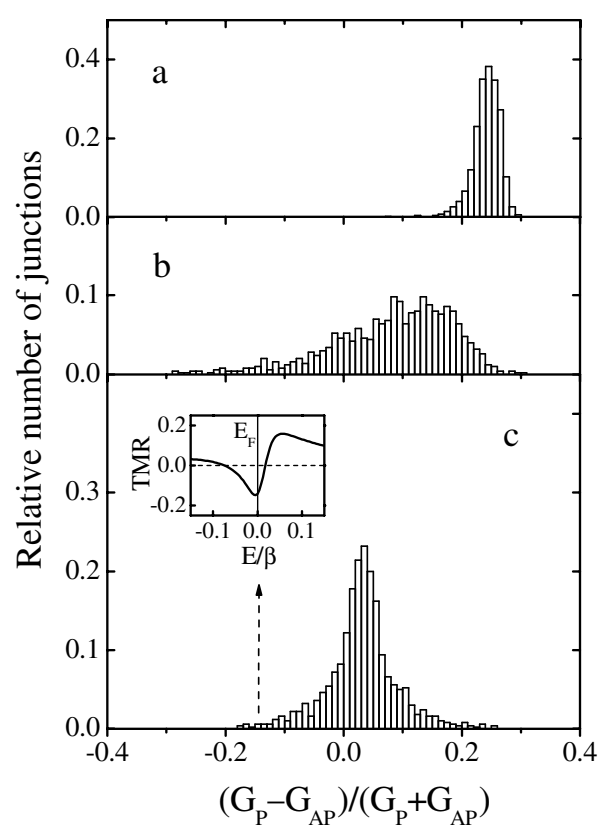

FIG. 3. Calculated distribution of TMR for various values of disorder $\gamma$ and inelastic scattering $\delta$ : (a) $\gamma=3 \beta, \delta=0$; (b) $\gamma=$ $4 \beta, \delta=0$; (c) $\gamma=4 \beta, \delta=0.01 \beta$. The inset of Fig. 3(c) shows TMR versus energy for a nanojunction with $\mathrm{TMR}=-0.14$ at $E=0$. 
structure of the barrier is contained in spin-dependent self-energies $\Sigma_{1,2}^{\uparrow, \downarrow}$. We parametrize the self-energies to the DOS of the electrodes, $\Sigma_{1,2}^{\uparrow, \downarrow}=-i \pi \beta^{2} \rho_{1,2}^{\uparrow, \downarrow}$, in the spirit of the model used in Ref. [22]. This allows introducing the spin polarizations of the electrodes, which in the calculations are taken to be $P=P_{1}=P_{2}=0.6$ - a representative value characterizing $\mathrm{Co}$ and $\mathrm{Ni}$ ferromagnets [23]. We include in our calculation inelastic scattering using a model of Ref. [24]. According to this model, each atomic site of the structure is connected to a "scattering" electrode that serves as a phase-breaking scatterer, thereby introducing an incoherent component to the overall current flow. Inelastic scattering is determined by the selfenergies of the scattering electrodes, parametrized so that $\Sigma_{S}=i \delta$, where $\delta$ is a parameter [22].

Figure 3 shows a calculated distribution of TMR for various values of disorder $\gamma$ and inelastic scattering $\delta$. As is seen from Fig. 3(a), for $\gamma=3 \beta$ and $\delta=0$, the TMR ratio peaks at values about 0.25 which is less than the value of $P^{2}=0.36$ expected for a perfect junction. No inverse TMR is present in this case due to a relatively low degree of disorder, which does not broaden the conduction band sufficient for creating localized states at the Fermi energy. The distribution of TMR dramatically changes when $\gamma=4 \beta$ and disorder generates localized states lying close to the $E_{F}$. As is seen from Fig. 3(b), this leads to a broad distribution of TMR that displays both positive and negative values. The negative values extend down to -0.3 approaching the minimal possible value of -0.36 according to Eq. (3). As is evident from Fig. 3(c), inelastic scattering narrows the distribution shifting the histogram maximum towards zero and making the distribution more symmetrical. The negative TMR values remain in the presence of inelastic scattering. The calculated energy dependence of TMR for a particular disorder configuration that gives TMR $=-0.14$ at $E=0$ reveals that the inverse TMR is indeed the consequence of resonant tunneling. As is seen from the inset of Fig. 3(c), the TMR is negative around the resonant energy, whereas it is positive everywhere else.

A comparison of the calculated distribution of TMR [Fig. 3(c)] with the experimental one (Fig. 1) demonstrates similarity in the width and the shape, as well as in the position of the maximum lying around 0.03 (if we disregard the experimental peak at small values below 0.01 on the grounds explained above). This agreement is obtained by fitting only two parameters, $\gamma$ and $\delta$. The obtained magnitude of inelastic scattering, $\delta=0.01 \beta \approx$ $0.015 \mathrm{eV}$, corresponds to a characteristic time $\tau=\hbar / \delta \approx$ $0.04 \mathrm{ps}$, which is comparable to the dephasing time in low-mobility semiconductors at low temperatures that varies from 0.1 to $1 \mathrm{ps}$ [25]. The correct magnitude of disorder parameter $\gamma$ is supported by similar values of the density of localized states at the $E_{F}$ obtained from our calculations and in the experiments. Indeed, for $\gamma=4 \beta$ (and $\beta \approx 1.5 \mathrm{eV}$ ), we find that the averaged DOS at $E_{F}$ is about $5 \times 10^{-3}$ atom ${ }^{-1} \mathrm{eV}^{-1}$. This value is similar to that deduced from Mott-Schottky analysis at ambient temperature. In first approximation, a density of carriers $n=10^{25} \mathrm{~m}^{-3}\left(10^{-4}\right.$ atom $\left.^{-1}\right)$ within a bandwidth of $k T \approx 0.025 \mathrm{eV}$ corresponds to the DOS of $4 \times 10^{-3}$ atom $^{-1} \mathrm{eV}^{-1}$.

In conclusion, we have shown that resonant tunneling can invert TMR in magnetic tunnel junctions. This phenomenon was observed in $\mathrm{Ni} / \mathrm{NiO} / \mathrm{Co}$ nanowire junctions of a small area and was explained in terms of disorder-driven statistical variations of TMR with a finite probability of inversion due to resonant tunneling. Our results indicate that the specifics of atomic arrangement in magnetic nanojunctions have a considerable impact on spin-dependent transport.

This research was supported by NSF (DMR-0203359 and DMR-9874657), ONR (N00140210610), and the Nebraska Research Initiative.

[1] J.S. Moodera et al., Annu. Rev. Mater. Sci. 29, 381 (1999); E. Y. Tsymbal et al., J. Phys. Condens. Matter 15, R109 (2003).

[2] M. Julliere, Phys. Lett. 54A, 225 (1975).

[3] S. Maekawa and U. Gäfvert, IEEE Trans. Magn. 18, 707 (1982).

[4] J. C. Slonczewski, Phys. Rev. B 39, 6995 (1989).

[5] S. Zhang and P. M. Levy, Eur. Phys. J. B 10, 599 (1999).

[6] E. Y. Tsymbal and D. G. Pettifor, Phys. Rev. B 58, 432 (1998).

[7] E. Y. Tsymbal and D. G. Pettifor, J. Phys. Condens. Matter 9, L411 (1997).

[8] Ph. Mavropoulos et al., Phys. Rev. Lett. 85, 1088 (2000).

[9] W. H. Butler et al., Phys. Rev. B 63, 54416 (2001).

[10] R. Jansen and J. S. Moodera, Appl. Phys. Lett. 75, 400 (1999).

[11] P. LeClair et al., Phys. Rev. Lett. 86, 1066 (2001).

[12] J. M. De Teresa et al., Phys. Rev. Lett. 82, 4288 (1999); Science 286, 507 (1999).

[13] R. Meservey and P. M. Tedrow, Phys. Rep. 238, 173 (1994).

[14] M. Sharma et al., Phys. Rev. Lett. 82, 616 (1999).

[15] B. Doudin et al., Phys. Rev. Lett. 79, 933 (1997).

[16] A. Sokolov et al., MRS Proc. 674, T5.9.1 (2001).

[17] G. Barral et al., Electrochim. Acta 40, 2815 (1995).

[18] W. Wernsdorfer et al., Phys. Rev. Lett. 77, 1873 (1996).

[19] T. Miyazaki and N. Tezuka, J. Magn. Magn. Mater. 151, 403 (1995).

[20] R. Landauer, IBM J. Res. Dev. 32, 306 (1988); M. Büttiker, IBM J. Res. Dev. 32, 317 (1988).

[21] G. A. Sawatzky and J.W. Allen, Phys. Rev. Lett. 53, 2339 (1984).

[22] E. Y. Tsymbal et al., Phys. Rev. B 66, 073201 (2002).

[23] D. J. Monsma and S. S. P. Parkin, Appl. Phys. Lett. 77, 720 (2000).

[24] M. Büttiker, Phys. Rev. B 33, 3020 (1986).

[25] J. J. Lin and J. P. Bird, J. Phys. Condens. Matter 14, R501 (2002). 